> L'appareil musculosquelettique (AMSQ) est primordial pour effectuer nos tâches quotidiennes et professionnelles, et interagir socialement avec notre entourage. Les pathologies de I'AMSP ont un retentissement important sur notre fonctionnement normal. II n'est donc pas étonnant que les pathologies de l'AMSO arrivent en tête dans les statistiques mondiales relatives à l'absentéisme professionnel ou aux coûts sociétaux liés à la prise en charge des patients qui en sont atteints. L'AMS $\rho$ est aussi impliqué dans des pathologies lourdes du système nerveux central, telles que la paralysie cérébrale (PC), dont l'étiologie complexe complique l'interprétation des signes cliniques et l'obtention d'un consensus sur la mise en place d'un suivi clinique. Le projet ICT4Rehab a développé une plateforme technologique intégrée proposant des outils qui permettent une gestion et une visualisation plus aisées des informations cliniques liées à l'AMSQ dans le cadre de la PC. Cette plate-forme est ouverte à tout centre clinique intéressé. <

Le monde clinique doit parfois faire face à des pathologies dont l'étiologie complexe pose des problèmes d'interprétation. De nombreux cliniciens sont dès lors demandeurs de nouveaux outils qui facilitent l'interprétation des signes cliniques présentés par des patients souffrant de telles pathologies et qui permettent une meilleure intégration des connaissances disponibles [1]. Cet article rapporte le travail multidisciplinaire mené lors de la réalisation du projet ICT4Rehab (www.ict4rehab.org/users); l'objectif de ce dernier est le développement d'une plate-forme technologique intégrée d'aide à la gestion de données cliniques, à la visualisation de ces dernières et à la décision clinique dans le cadre de la paralysie cérébrale (PC). Le but ultime de cette plate-forme est d'offrir la possibilité d'effectuer des études multicentriques grâce à un par-

\section{Une plate-forme technologique liée à la paralysie cérébrale}

\section{Le projet ICT4Rehab}

Serge Van Sint Jan ${ }^{1}$, Vanessa Wermenbol ${ }^{2}$, Patrick Van Bogaert ${ }^{2}$, Kaat Desloovere ${ }^{3}$, Marc Degelaen ${ }^{4,5}$, Bernard Dan ${ }^{4}$, Patrick Salvia ${ }^{1,6}$, Els Ortibus ${ }^{7}$, Bruno Bonnechère ${ }^{1}$, Yann-Aël Le Borgne ${ }^{8}$, Gianluca Bontempi ${ }^{8}$, Stijn Vansummeren', Victor Sholukha ${ }^{1}$, Fédor Moiseev ${ }^{1}$, Marcel Rooze ${ }^{1,10}$

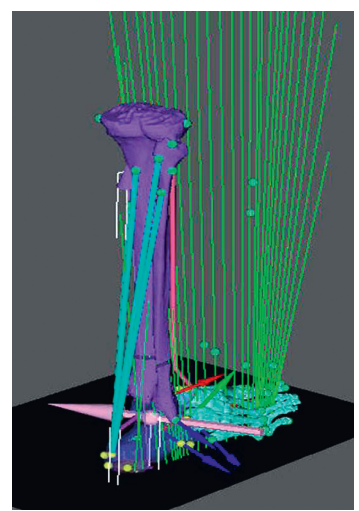

tage des données disponibles et de mieux interpréter les relations entre les signes cliniques observés, ceci afin, à plus long terme, d'améliorer le suivi clinique des patients. Afin de mieux comprendre les problèmes pratiques de la mise en place d'une telle plate-forme multicentrique partagée, il faut se rappeler que les stratégies cliniques liées à une atteinte telle que la paralysie cérébrale doivent faire face à la complexité anatomique et physiologique de l'appareil musculosquelettique (AMSO) et des troubles neuromusculaires associés [2].

\section{L'appareil musculosquelettique}

'Laboratoire d'anatomie, biomécanique et organogenèse, CP 619, faculté de médecine, Université libre de Bruxelles (ULB), 808 route de Lennik, 1070 Bruxelles, Belgique ;

${ }^{2}$ Service de neuropédiatrie, hôpital Erasme, ULB, Bruxelles, Belgique ;

${ }^{3}$ Center of evaluation of motor functions rehabilitation, Leuven hospital, Katholiek Universiteit Leuven (KUL), Belgique ;

${ }^{4}$ Neurologie pédiatrique, centre hospitalier universitaire des enfants Reine Fabiola, Bruxelles, Belgique;

${ }^{5}$ Hôpital de réadaptation Inkendaal, Vlezenbeek, Belgique; ${ }^{6}$ Centre d'évaluation fonctionnelle, ULB, Bruxelles, Belgique;

${ }^{7}$ Neurologie pédiatrique, Leuven hospital, KUL, Belgique ; ${ }^{8}$ Machine learning group, faculté des sciences, ULB, Bruxelles, Belgique;

${ }^{9}$ Computer and decision engineering department, École polytechnique, ULB, Bruxelles, Belgique ;

${ }^{10}$ Service d'orthopédie, hôpital Erasme, ULB, Bruxelles, Belgique. sintjans@ulb.ac.be
L'AMSQ est relativement méconnu du grand public. Pourtant, ses pathologies sont souvent invalidantes et reconnues comme étant l'une des causes principales de perte de revenus pour les sujets atteints, qui deviennent incapables de mener à bien une vie professionnelle 


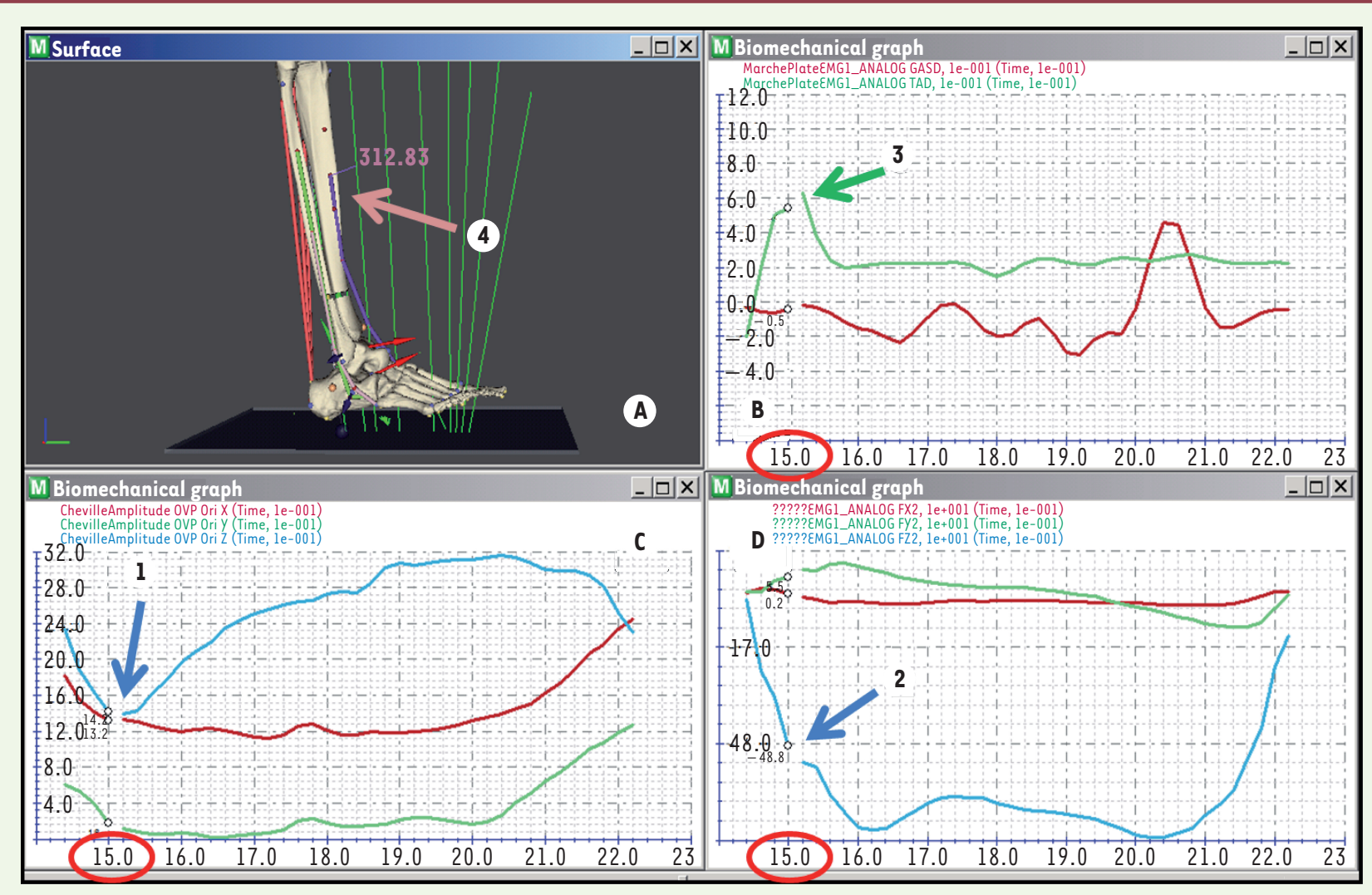

Figure 1. Outil de visualisation avancée des données avec le logiciel lhpFusionBox. A. Affichage graphique pour le contrôle de qualité des examens. B. Examen électromyographique (ici des muscles gastrocnémien latéral et tibial antérieur, respectivement en rouge et en vert). C. Courbes de mouvements (ici de la cheville, la flexion/extension est représentée par la courbe bleue). $\boldsymbol{D}$. Courbes des forces de réaction du sol. Ce genre d'interface permet de comparer les informations médicales disponibles grâce à un alignement spatio-temporel des données. Par exemple, au temps 15 (cercle rouge), la comparaison des graphiques indique que (1) la flexion plantaire totale de la cheville est presque atteinte, (2) sans que le maximum de force externe fournie par le sol soit atteint, de plus (3), le muscle tibial antérieur est actif, et (4) il a pratiquement atteint sa longueur maximale. De nombreuses autres informations peuvent être ainsi visualisées avant d'être intégrées dans la base de données.

normale [3]. La physiologie complexe de l'AMSP reste encore mal comprise bien qu'elle soit largement basée sur des principes physiques établis. Ceci est dû au fait que l'architecture anatomique de l'AMSP n'est encore que partiellement détaillée, ce qui rend le suivi clinique des pathologies associées à l'AMS $Q$ souvent difficile.

L'AMS $Q$ comporte plusieurs centaines de composants (os, articulations, muscles, etc.) ayant chacun un rôle spécifique ${ }^{1}[4]$. Le rôle relatif de ces composants n'est toujours pas clairement compris. Cette complexité s'accroît encore si l'on considère les dépendances physiologiques de I'AMS $\rho$ avec d'autres systèmes anatomiques, tels que les systèmes nerveux central (SNC) et périphérique (SNP). Une perturbation de ces dépendances peut entraîner un large éventail de dysfonctionnements neuromusculaires, tels qu'une paralysie partielle ou totale, une faiblesse musculaire, une spasticité musculaire ou encore une perte de coordination motrice [5]. Selon l'intensité de ces perturbations, l'indé-

${ }^{1}$ Citons les 103 paires d'os (lieux d'insertions des muscles et ligaments); les articulations (permettant le mouvement); et les muscles (plus de 300 paires, dont la contraction permet le mouvement). pendance fonctionnelle des sujets se réduira de façon proportionnelle. Le patient risque alors de s'isoler, de rester à domicile, ce qui entraînera des troubles sociaux et psychologiques. Ces signes cliniques sont observables dans plusieurs catégories de patients atteints de maladies neuromusculaires liées au système nerveux central (paralysie cérébrale et lésions de la moelle épinière), aux motoneurones (amyotrophie spinale), au système nerveux périphérique (maladie de Charcot-Marie-Tooth, myasthénie grave), ou encore aux muscles (dystrophie de Duchenne) [2]. Pour réduire les conséquences invalidantes de ces pathologies, un des défis actuels relevés par les équipes thérapeutiques est de mieux comprendre l'origine et la prépondérance relative des différents signes cliniques observés chez leurs patients. La complexité de I'AMSO et de ses relations rend difficile le diagnostic précis relatif aux problèmes spécifiques d'un patient. Le développement d'outils d'aide à la clinique 
est donc justifié. Le projet ICT4Rehab s'adresse plus particulièrement aux problèmes spécifiques de la paralysie cérébrale.

\section{La paralysie cérébrale}

La paralysie cérébrale (aussi connue sous le nom d'infirmité motrice cérébrale) est un syndrome caractérisé principalement par des troubles moteurs non progressifs associés à des lésions primaires apparaissant à un stade précoce du développement du cerveau [6]. Sa prévalence est d'environ 1/500 naissances viables. La lésion primaire cérébrale, irréversible, entraîne un tableau compliqué de signes cliniques secondaires variables d'un patient à l'autre [7]. Outre les dysfonctionnements associés directement à l'AMSQ (voir section précédente), le patient atteint de paralysie cérébrale peut présenter des signes cliniques secondaires supplémentaires: troubles cognitifs, sensitifs et visuels, épilepsie ou encore incontinence urinaire [7]. La localisation et la nature de la lésion primaire sont aussi variables et justifient la répartition des patients en sous-groupes selon le type (spasticité, ataxie, dyskinésie) et la distribution (diplégie, hémiplégie, tétraplégie) de l'atteinte. Le travail thérapeutique a pour objectif essentiel de diminuer l'ampleur des signes cliniques secondaires [5]. La compréhension de ces derniers lorsqu'ils sont présentés par un patient particulier est donc primordiale. Malheureusement, le nombre et l'intensité de ces signes cliniques secondaires sont variables d'un patient à l'autre, rendant délicate la mise en place d'une stratégie thérapeutique individuelle. Afin de mieux en comprendre l'importance relative chez un patient particulier, de nombreux examens cliniques (explicités dans la section suivante) sont disponibles.

\section{Trop d'informations cliniques disponibles?}

Les informations cliniques liées à la paralysie cérébrale sont très nombreuses. Elles peuvent être regroupées en plusieurs catégories selon leur nature et leur modalité d'acquisition : anamnèse du patient, examens cliniques, examens fonctionnels quantitatifs et examens complémentaires (Tableau I) [5]. Le choix de l'utilisation de ces informations dépend des habitudes locales des équipes cliniques ou des ressources matérielles disponibles. Paradoxalement, cette pléthore relative d'informations cliniques disponibles dans le cadre du suivi d'un patient atteint de paralysie cérébrale génère plusieurs difficultés. Le premier problème est lié à la mise en relation des informations cliniques formant le dossier d'un patient particulier avec celles concernant des patients similaires. Par exemple : une série de patients ont reçu des injections de drogues myorelaxantes (par exemple, toxine botulinique) dans certains de leurs muscles afin de réduire la spasticité observée et espérer ainsi faciliter leur marche. L'analyse du mouvement [8] effectuée sur ces patients permet de quantifier leur mobilité articulaire pendant la marche avant et après injection. Un nouveau patient présente un schéma de marche légèrement dissemblable par rapport au groupe de référence. Peut-on mettre en relation les informations contenues dans le dossier des différents patients (muscles injectés, volume injecté, fréquence, quantification de la mobilité articulaire) avec celles du nouveau patient afin de tirer des conclusions statistiques quant au meilleur volume d'injection pour ce dernier? Une telle mise en relation statistique entre les observations quantifiées et l'acte clinique (ici, l'injection de drogue) reste difficile à cause de l'hétérogénéité des résultats chez les patients analysés. Il est donc primordial que le contenu du dossier des patients soit bien défini afin de garantir une meilleure compréhension des relations qui existent entre les gestes thérapeutiques disponibles et les données cliniques obtenues.

La seconde difficulté est liée à la pléthore d'informations mentionnée ci-dessus qui a pour conséquence que l'interprétation des signes cliniques secondaires est souvent différente d'un centre clinique à l'autre. II n'existe pas actuellement d'outils statistiques permettant de suivre longitudinalement et objectivement un patient à partir des observations disponibles. L'interprétation du tableau clinique d'un patient est ainsi surtout dépendante de l'expérience de l'équipe soignante locale. II n'est donc pas rare qu'un même patient puisse se voir proposer des traitements parfois divergents s'il s'adresse à différents centres cliniques. Outre le fait de pouvoir créer une certaine confusion dans l'esprit de ce patient, ces différences freinent l'établissement de procédures cliniques standardisées.

On le voit, un effort multidisciplinaire est entièrement justifié afin de pouvoir faciliter la prise en charge du patient atteint de paralysie cérébrale via une meilleure exploitation scientifique des informations cliniques contenues dans son dossier.

\section{Vers une facilitation de la gestion et de l'exploitation clinique du dossier du patient}

Le projet ICT4Rehab est organisé autour d'un consortium multidisciplinaire dont le but est d'offrir une plateforme technologique d'aide à l'exploitation des informations disponibles en clinique. Ses facettes sont brièvement expliquées dans la suite de l'article.

\section{Validation des informations fournies}

Le fait de collecter une information clinique est par essence un acte similaire à une prise de mesure scientifique. Ces informations cliniques doivent donc être obtenues en respectant les règles de l'art. Une condition fondamentale pour qu'une mesure soit fiable est que le protocole permettant son acquisition soit validé $[9,10]$. Le non-respect de cette règle diminue la valeur clinique des mesures acquises obtenues et, dès lors, l'intérêt de leur exploitation pour le suivi des progrès d'un patient. Ceci est valable pour toutes les modalités disponibles (Tableau 1). Pourtant, de nombreux cliniciens oublient 


\begin{tabular}{|c|c|c|}
\hline Catégorie & Nom de l'information (= donnée clinique) & $\begin{array}{c}\text { À propos de l'examen générant } \\
\text { l'information }\end{array}$ \\
\hline \multirow[b]{3}{*}{ Anamnèse } & Données personnelles & Informations générales (nom, sexe, date de naissance, etc.) \\
\hline & $\begin{array}{l}\text { Âges : à la naissance (gestationnel pour les enfants } \\
\text { prématurés), position assise, marche, etc. }\end{array}$ & Moments clefs du développement \\
\hline & $\begin{array}{l}\text { Historique thérapeutique : médication, attelles, } \\
\text { kinésithérapie, drogues myorelaxantes, chirurgie } \\
\text { orthopédique, etc. }\end{array}$ & $\begin{array}{l}\text { Suivi clinique du patient ; permet de comparer cet } \\
\text { historique avec le résultat des tests cliniques et } \\
\text { fonctionnels (voir ci-dessous) pour juger de l'efficacité de } \\
\text { la prise en charge clinique }\end{array}$ \\
\hline \multirow{4}{*}{ Examens cliniques } & $\begin{array}{l}\text { Évaluation quantitative de la fonction motrice : tests } \\
\text { cliniques tels que le Gross motor function classification } \\
\text { system (GMFCS), le Manual ability classification system } \\
\text { (MACS), l'index de consommation énergétique, etc. }\end{array}$ & Quantification de certains aspects fonctionnels [5] \\
\hline & $\begin{array}{l}\text { Évaluation de la spasticité et de la force musculaire: } \\
\text { tests cliniques tels que l'échelle d'Ashworth (test de } \\
\text { spasticité), le muscle rating scale (force musculaire), etc. }\end{array}$ & Quantification de la fonction musculaire [5] \\
\hline & $\begin{array}{l}\text { Évaluation de l'amplitude articulaire: flexion/extension } \\
\text { de la hanche avec le genou tendu et fléchi, etc. }\end{array}$ & Évaluation de l'amplitude des articulations impliquées [5] \\
\hline & Morphologie osseuse : antéversion fémorale, patella alta, etc. & Objectivation des déformations osseuses [5] \\
\hline \multirow{4}{*}{$\begin{array}{l}\text { Examens } \\
\text { fonctionnels } \\
\text { quantitatifs }\end{array}$} & Analyse de mouvements & $\begin{array}{l}\text { Quantification objective de la fonction d'un membre } \\
\text { pendant une tâche particulière (un membre supérieur } \\
\text { pendant la prise d'objets, les membres inférieurs pendant la } \\
\text { marche) [8] }\end{array}$ \\
\hline & Électromyographie & $\begin{array}{l}\text { Indication de l'activité musculaire pendant } \\
\text { l'accomplissement d'une tâche particulière. Souvent } \\
\text { combinée à l'analyse de mouvements }\end{array}$ \\
\hline & Tests ambulatoires ou de performance fonctionnelle & $\begin{array}{l}\text { Le patient est sollicité pour faire une tâche spécifique (par } \\
\text { exemple, prendre un objet d'une certaine taille, marcher sur } \\
\text { une certaine distance, etc.) }\end{array}$ \\
\hline & Pédobarographie & Analyse des pressions plantaires mesurées \\
\hline \multirow[b]{2}{*}{$\begin{array}{l}\text { Examens } \\
\text { complémentaires }\end{array}$} & Imagerie médicale & $\begin{array}{l}\text { Visualisation de la morphologie du patient (squelette et } \\
\text { cerveau) }\end{array}$ \\
\hline & Visualisation avancée par fusion de données (Figure 1) & $\begin{array}{l}\text { Estimation de paramètres physiologiques non directement } \\
\text { mesurables sur le patient (par exemple, la variation de } \\
\text { longueur des muscles). Cet examen est récent et se trouve } \\
\text { encore dans une phase de validation clinique }\end{array}$ \\
\hline
\end{tabular}

Tableau I. Exemples-types d'informations cliniques trouvées au sein du dossier d'un patient atteint de paralysie cérébrale. Ce tableau n'est pas exhaustif ; le contenu d'un tel dossier présente d'importantes différences d'un centre clinique à l'autre. II n'existe actuellement aucune manière d'intégrer facilement ces informations au sein d'un rapport clinique [5].

cet aspect. Le consortium ICT4Rehab insiste donc pour que des règles de bonnes pratiques soient strictement adoptées afin d'assurer des conditions optimales pour la collecte des informations en clinique et garantir leur qualité. L'adoption de ces règles est une condition sine qua non pour que des outils tels que ceux développés par le projet ICT4Rehab soient réellement efficaces et utiles. De telles règles doivent être mises en place par des autorités compétentes (par exemple, les associations professionnelles concernées) à partir de recommandations déjà disponibles dans la littérature $[5,11,12]$. Des démarches récentes 


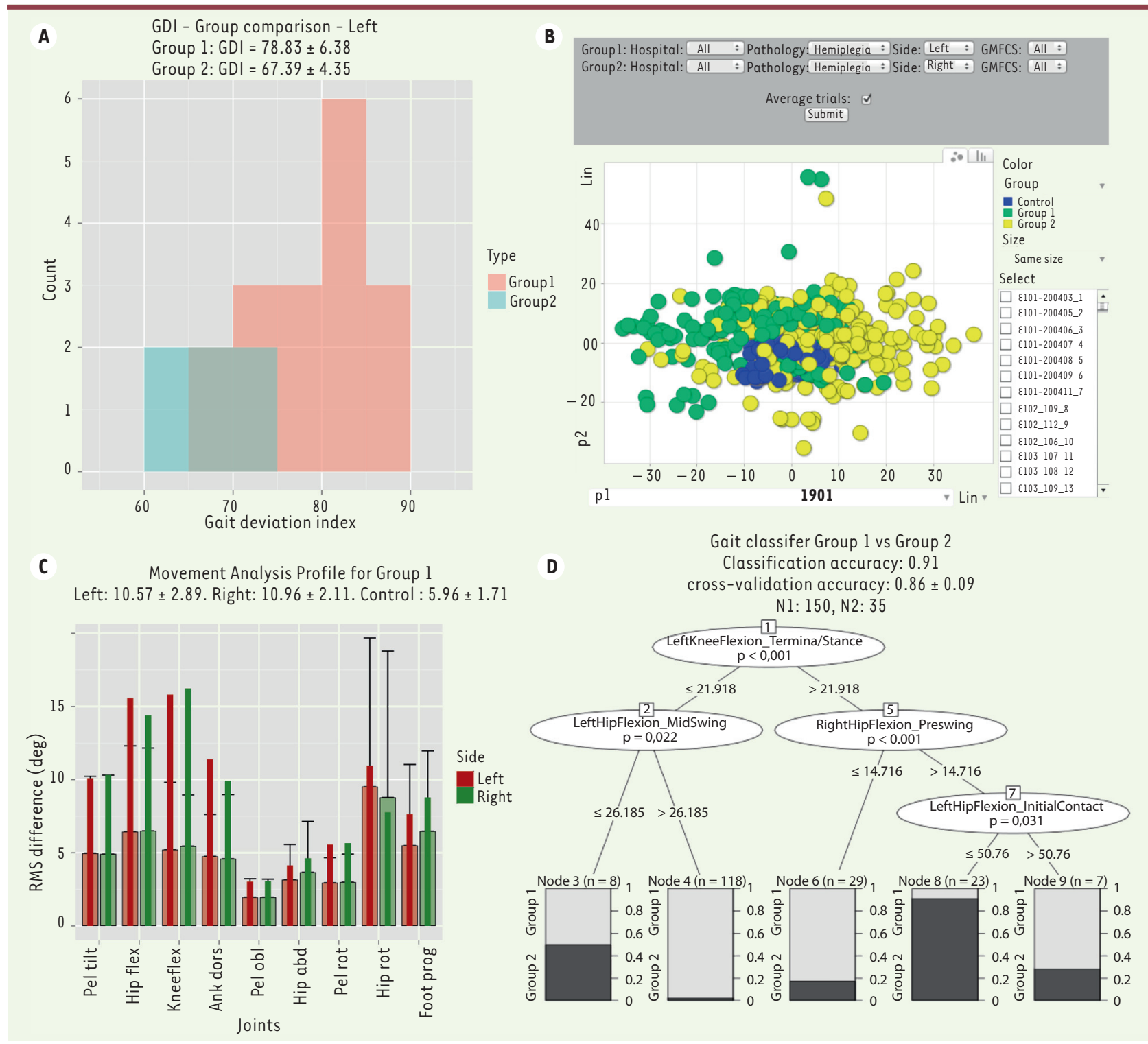

Figure 2. Exemple de résultats obtenus à partir de la fouille de données. A. Histogrammes comparant deux groupes de patients (GMFCS II et III [Gross motor function classification system], en rose et bleu, respectivement) en termes de Gait deviation Index (GDI). B. Analyse en composante principale des données de cinématiques et affichage des populations contrôle (en bleu), hémiplégie gauche (en vert) et hémiplégie droite (en jaune). C. Movement analysis Profile (MAP) pour un groupe de patients ayant un GMFCS III. D. Arbre de décision basé sur les données de cinématiques permettant de discriminer les patients GMFCS II des patients GMFCS III. Ce genre d'outil permet, rapidement et objectivement, de comparer les données d'un patient avec celles d'autres groupes de patients.

ont d'ailleurs été effectuées afin de promouvoir la mise en place de contrôles garantissant la qualité des mesures prises lors de l'analyse de la marche $[13,14]$. Il faut espérer que ces directives soient adoptées par la communauté clinique afin de standardiser les protocoles utilisés. La validation est liée à la reproductibilité et aux erreurs de mesure, cette dernière comprenant les notions de justesse et de précision $[9,10]$. La reproductibilité implique qu'une même mesure effectuée par plusieurs personnes aboutisse à des résultats similaires; ceci garantit que les signes cliniques d'un patient soient quantifiés de la même manière quel que soit le clinicien faisant le suivi clinique. La justesse s'évalue par l'écart d'une mesure par rapport à la vraie valeur du phénomène observé. La précision permet de définir l'intervalle des valeurs mesurées. Notons qu'une mesure dont la reproductibilité est bonne, mais la justesse faible, peut quand même avoir un intérêt en clinique : elle ne permettra pas de connaître l'état d'un patient de façon juste, mais des mesures répétées dans le temps permettront d'obtenir des informations sur l'évolution de son état. En revanche, une mesure non reproductible est à proscrire absolument. 


\section{Frein}

I. Vie privée

du patient (identité, Éthique et légal

confidentialité, etc.)

II. Sécurité

des données

Éthique et légal

IIla. Saisie multiple

des données

Éthique et légal

Illb. Saisie multiple

des données

Humain

IV. Comité d'éthique Éthique et légal
et vie privée

$\ln (\ln )$

V. Propriétés
des données

Légal

VI. Accessibilité

des données

Humain

des don

VII. Propriétés
des résultats

VIII. Qualité

des données

et standards

Scientifique

\section{Pérennité}

des données

Scientifique

\section{Pérennité}

de la base

de données

\section{Problème $(P)$ et solution $(S)$}

P: I'accès aux données partagées doit assurer que l'identité du patient ne peut être retrouvée

$S$ : l'identité d'un patient peut être encodée, voir encryptée, sans que celle-ci ne soit présente physiquement sur le support partagé. Ce type d'encodage doit se faire dans le respect des lois européennes sur la protection de la vie privée et de l'anonymat du patient [17]

P: copiage illégal des données

S: la technologie existe pour augmenter la sécurité des structures partagées et diminuer l'intrusion au sein du système, par exemple par utilisation de consoles de travail dédiées uniquement à l'accès à la base de données [18]

P: tant que les freins I et II ne sont pas résolus, la gestion des hôpitaux refusera que le dossier des patients soit conservé uniquement sur un serveur partagé localisé en dehors de l'hôpital ; une double saisie des données est donc requise pour pouvoir profiter des bénéfices d'un système partagé.

$S$ : il existe des technologies dites de décentralisation de données permettant un partage virtuel de certaines données qui restent dans l'hôpital responsable [18]

P: si le problème Illa n'est pas résolu, certains cliniciens trouveront qu'un second encodage est contre-productif $S$ : il est important que ces cliniciens réalisent l'importance d'une structure de données partagées pour pouvoir espérer améliorer leur compréhension de la complexité de la paralysie cérébrale, et ainsi améliorer la qualité de vie de leurs patients

P: le partage de données fait l'objet de règles strictes et doit recevoir l'aval des comités d'éthique de chacun des hôpitaux concernés, ainsi que l'aval des patients

$S$ : au minimum les points précités doivent être résolus. Dans le cadre d'un registre national, un comité d'éthique national doit superviser la demande (par exemple, la Commission pour la protection de la vie privée en Belgique [19]). Les patients concernés doivent être informés du devenir des examens médicaux les concernant et doivent donner leur consentement éclairé sur ce devenir

$\mathbf{P}$ : les données restent la propriété de l'institution ayant produit les examens médicaux, y compris toute copie faite en dehors de cette institution

$S$ : ceci est réglé par un contrat entre l'institution et le gestionnaire de la base de données. Au niveau de la base de données, l'institution peut retirer à tout moment ses données ou en réduire l'accessibilité (voir point VI dans ce tableau). Le propriétaire de chaque donnée est clairement identifié au niveau de la base de données

$\mathbf{P}$ : certaines institutions ne veulent pas partager leurs données avec n'importe qui (pour des raisons internes à respecter)

S: l'institution décide elle-même les groupes d'utilisateurs pouvant accéder à ses données via une interface simple associée à la base de données partagée

P: les outils associés à la base de données vont générer de nouvelles conclusions susceptibles d'être publiées. À qui appartiennent ces résultats?

$S$ : ceci est difficile à définir à l'avance. Chaque utilisateur s'engage formellement, quand il s'inscrit comme utilisateur, à contacter le(s) propriétaire(s) des données en cas de valorisation des résultats

$\mathrm{P}$ : la qualité des données introduites dans la base de données doit répondre à certains standards scientifiques (voir section Validation des informations fournies) afin de garantir des résultats statistiques de qualité.

S: des outils associés à la base de données permettent de comparer les données à un standard acceptable ; le cas échéant des données dont la validation n'est pas satisfaisante peuvent être éliminées du traitement statistique.

P: étant donné que les standards utilisés pour le point VIII peuvent évoluer, certaines données présentes dans la base de données peuvent devenir obsolètes parce qu'elles ne répondent plus aux nouveaux standards.

$S$ : voir point VIII de ce tableau.

P: la survie à long terme d'une plate-forme technologique est directement liée au financement de moyens permettant sa maintenance.

S: vu la nature de l'information contenue dans la base de données, la solution la plus logique serait qu'une telle maintenance soit assurée par des structures supra-hospitalières (sécurité sociale). Une maintenance basée sur des financements à court terme est un point de démotivation pour des utilisateurs cliniques potentiels qui hésiteront à s'impliquer dans un projet dont l'avenir est incertain.

Tableau II. Freins médico-légaux, humains et scientifiques réduisant l'utilisation de systèmes partagés en clinique, et solutions possibles. $P$ : description du problème $; S$ : solution envisageable. 
En clinique, certains tests, comme ceux basés sur des scores simples, n'ont pas une bonne réputation au niveau de leur validité scientifique [5]. Pourtant, ils sont encore très utilisés en clinique, ce qui soulève des questions quant à l'interprétation et la validité de ces informations obtenues d'un clinicien à l'autre. D'autres modalités, comme les examens fonctionnels quantitatifs (Tableau I), basées sur I'utilisation d'outils de mesure validés, sont reconnues comme étant plus objectives $[5,8]$. II incombe aux cliniciens de sélectionner les tests qui garantissent à leurs patients le meilleur suivi longitudinal. Dans le cadre d'études multicentriques, le choix des tests pertinents est d'autant plus critique, il doit garantir que les tests retenus soient reproductibles.

\section{Base de données}

Un nombre aussi important d'informations disparates requiert une base de données adaptée en conséquence. La base de données développée par le projet ICT4Rehab est capable de stocker, dans un format normalisé, la plupart des données relatives à l'anamnèse, aux examens cliniques et à certains examens fonctionnels quantitatifs, tels que l'analyse de mouvements et l'électromyographie (Tableau I). Ce souhait d'exhaustivité devrait permettre à des groupes cliniques ayant des habitudes locales partiellement différentes de pouvoir réaliser des études statistiques multicentriques à partir des données qu'ils ont en commun. Le formatage de l'encodage de la plupart des tests cliniques au sein de la base de données est facilité par le fait que ces tests sont de conception simple. Pour les examens fonctionnels quantitatifs, le format standard C3D (www.C3D.org) a été adopté. Une interface web garantit que les utilisateurs peuvent accéder à la base de données sans recours à un logiciel particulier, un navigateur standard étant suffisant. L'anonymat des patients est assuré. De plus, les utilisateurs peuvent spécifier le niveau de partage des données placées sous leur responsabilité : ces données peuvent être, soit conservées comme privées, soit partagées par plusieurs groupes prédéfinis d'utilisateurs dans le cadre d'études multicentriques. Enfin, la base de données est directement accessible à des outils statistiques sophistiqués (voir plus loin).

Notons qu'actuellement, la plate-forme requiert une double saisie des données par l'utilisateur (une saisie locale pour les archives internes de l'institution où se fait le suivi du patient, et une saisie au travers de l'interface de la base de données). Ceci peut être un facteur démotivant pour l'utilisateur clinique modal ne possédant que peu de temps dans sa pratique quotidienne. L'interface d'accès à la base de données du projet ICT4Rehab a dès lors été conçue afin de minimiser le temps requis pour l'entrée des données. Notons que cette saisie supplémentaire devrait être largement compensée par les bénéfices apportés par une structure informatisée multicentrique mettant à disposition des outils statistiques portant sur un grand nombre de patients.

\section{Fouille de données}

L'avantage majeur d'une base de données formatée est la possibilité de traiter l'information disponible grâce à des outils statistiques avancés, dits de fouille de données. Dans le cadre du projet ICT4Rehab, ces outils ont pour objectif d'aider à l'exploitation des infor- mations contenues dans la base de données. Ainsi, un ensemble d'outils existe pour faciliter la visualisation des informations, leur comparaison multicentrique (par exemple, pour s'assurer que certaines données aient été collectées selon les critères susmentionnés de bonne pratique) et la détermination d'associations statistiquement significatives entre les informations disponibles (voir la section Trop d'informations cliniques disponibles?). Le développement de ces outils se fait en collaboration étroite avec des cliniciens associés au projet, afin d'identifier les questions pertinentes, et d'apporter les solutions algorithmiques les plus appropriées. Rappelons que la qualité des résultats de la fouille de données dépend fortement du niveau de validation des données disponibles. Les outils de comparaison de population et de différenciation sont d'ores et déjà accessibles à http://mlg.ulb. ac.be/ICT4Rehab_DM (Figure 2). Ces outils sont basés sur des algorithmes standard de fouille de données tels que le clustering, l'analyse en composante principale et les arbres de décisions [15, 16] (la version actuelle de l'interface ne permet que l'analyse de données en provenance de l'analyse quantifiée du mouvement; une extension à d'autres données cliniques est prévue dans le courant de l'année 2013). Ces résultats statistiques seront intégrés très bientôt dans des rapports cliniques qui sont en développement, en collaboration avec les partenaires cliniques du projet.

\section{Modélisation par fusion de données}

Toutes les données décrites ci-dessus sont mesurées directement sur le patient. Bien que nombreuses, elles ne fournissent pas encore toutes les réponses. D'autres informations importantes ne sont pas mesurables directement vu les limites de la technologie actuelle, comme par exemple l'excursion musculaire qui quantifie la longueur d'un muscle pendant un mouvement donné. Des techniques dites de «fusion de données » ont été récemment développées pour répondre à cette demande. La fusion consiste à la combinaison de données venant de modalités différentes [20]. De nombreux protocoles cliniques utilisant l'imagerie médicale y font recours. En revanche, les données relatives à I'AMSQ sont de nature très disparate et dès lors se prêtent moins bien à la fusion. De nouveaux développements scientifiques furent donc nécessaires dans le cadre du projet ICT4Rehab. De nouveaux algorithmes ont été développés et peuvent combiner des données hétérogènes acquises sur le patient (imagerie médicale, analyse de mouvements, pédobarographie) avec des données provenant de la recherche fondamentale. Le modèle obtenu (Figure 1) permet ainsi une meilleure 
interprétation des informations cliniques disponibles ainsi que l'estimation d'informations supplémentaires comme l'excursion musculaire. Ce développement est très récent et la méthodologie est actuellement au stade de validation clinique.

\section{Freins humains, scientifiques, éthiques et légaux}

La technologie proposée par ICT4Rehab en termes de gestion et de compréhension de données devrait aider le monde clinique à mieux comprendre la complexité de certaines pathologies, telles que la PC. Pourtant, des freins peuvent limiter l'adoption de cette technologie. Ces blocages sont liés à des facteurs éthiques et légaux, scientifiques, ou encore humains (Tableau II). La résolution de ces freins dépend surtout de la motivation des acteurs concernés. Les freins éthiques et légaux demandent de respecter des procédures administratives en place. Les freins scientifiques peuvent être résolus grâce à l'adoption de standard disponibles dans la littérature ; le problème ici est de trouver un standard acceptable par une majorité d'utilisateurs. L'aspect humain est peut-être plus difficile à résoudre : l'argument le plus persuasif étant que la disponibilité d'une large base de données devrait faire évoluer nos connaissances dans le domaine et finalement en faire bénéficier le patient qui reste le premier concerné. II faut évidemment que l'activité des acteurs cliniques impliqués soit valorisée, par exemple en les associant aux publications émergeant des études statistiques faites à partir de la base de données.

\section{Conclusions}

La prise en charge clinique de la PC reste un problème difficile en clinique. Ceci est dû au manque actuel d'outils adaptés à la gestion des informations cliniques, ainsi qu'à leur traitement, interprétation et échange multicentriques. De tels outils ont été développés au sein du projet ICT4Rehab. Ce dernier construit une plateforme technologique prenant en compte les problèmes cliniques actuels mentionnés dans cet article. La plateforme sera disponible à la fin mars 2013 pour tout clinicien intéressé à participer à l'organisation d'une étude multicentrique portant sur le suivi de patients paralysés cérébraux. $\diamond$

\section{SUMMARY}

A technological platform for cerebral palsy - The ICT4Rehab project The musculoskeletal system (MSS) is essential to allow us performing every-day tasks, being able to have a professional life or developing social interactions with our entourage. MSS pathologies have a significant impact on our daily life. It is therefore not surprising to find MSSrelated health problems at the top of global statistics on professional absenteeism or societal health costs. The MSS is also involved in central nervous conditions, such as cerebral palsy (CP). Such conditions show complex etiology that complicates the interpretation of the observable clinical signs and the establishment of a wide consensus on the best practices to adopt for clinical monitoring and patient follow-up. These elements justify the organization of fundamental and applied research projects aiming to develop new methods to help clinicians to cope with the complexity of some MSS disorders. The ICT4Rehab project (www.ict4rehab.org) developed an integrated platform providing tools that enable easier management and visualization of clinical information related to the MSS of CP patients. This platform is opened to every interested clinical centre. $\diamond$

\section{LIENS D'INTÉREิT}

Les auteurs déclarent n'avoir aucun lien d'intérêt concernant les données publiées dans cet article.

\section{REMERCIEMENTS}

Le projet ICT4Rehab est financé par la région bruxelloise via son agence d'aide à l'innovation Innovlris.

\section{RéFÉRENCES}

1. Tirosh 0, Baker R, McGinley J. GaitaBase: web-based repository system for gait analysis. Comput Biol Med $2010 ; 40: 201-7$.

2. Driscoll S, Skinner J. Musculoskeletal complications of neuromuscular disease in children. Phys Med Rehabil Clin N Am 2008 ; 19 : 163-94.

3. The Burden of musculoskeletal diseases in the United States: prevalence, societal and economic cost. http://www.boneandjointburden.org/.

4. Dalley A, Moore K, Agur A. Anatomie médicale : aspects fondamentaux et aspects cliniques. Bruxelles: De Boeck, 2011.

5. Brashear A, Elovic E. Spasticity: diagnosis and management. New York: Demos Medical, 2011.

6. Rosenbaum $P$, Paneth $\mathrm{N}$, Leviton $\mathrm{A}$, et al. The definition and classification of cerebral palsy. Dev Med Child Neurol 2007 ; 49 : 1-44.

7. Odding $\varepsilon$, Roebroeck M, Stam M. The epidemiology of cerebral palsy: incidence, impairments and risk factors. Disabil Rehabil 2006 ; 28 : 183-91.

8. Penneçot G. Marche pathologique de l'enfant paralysé cérébral. Montpellier : Sauramps Médical, 2009.

9. Gonzalez A, Herrador M. A practical guide to analytical method validation, including measurement uncertainty and accuracy profiles. Trends Anal Chem $2007 ; 26: 227-38$.

10. Dijkers M, Kropp G, Esper R, et al. Reporting on reliability and validity of outcome measures in medical rehabilitation research. Disabil Rehabil 2002 ; $16: 819-27$.

11. Wu G, Siegler S, Allard P, et al. ISB recommendation on definitions of joint coordinate system of various joints for the reporting of human joint motion. Part I: ankle, hip, and spine. J Biomech $2002 ; 35$ : 543-8.

12. Van Sint Jan S, Della Croce U. Identifying the location of human skeletal landmarks: why standardized definitions are necessary: a proposal. Clin Biomech 2005; $20: 659-60$.

13. Rouzel $\varepsilon$, Paucton $M$, Farges $G$, et al. Démarche qualité en laboratoire de recherche biomédicale : application pour l'analyse quantifiée de la marche (AQM). IRBM News $2012 ; 33: 86-9$.

14. Commission for motion laboratory accreditation. http://www.cmlainc.org.

15. Trevor J, Tibshirani R, Friedman J. The elements of statistical learning: data mining, inference and prediction. New York : Springer Verlag, 2009.

16. Wu X, Kumar V. The top ten algorithms in data mining. Boca Raton : Chapman and Hall CRC, 2009.

17. Directives $95 / 46 / \varepsilon C$ et $2001 / 20 / \varepsilon C$ du parlement européen.

18. De Wolf C. Integrating digital mammography in a decentralised breast cancer screening programme. Oxford, United Kingdom: International Cancer Screening Network, 2010.

19. Commission de la vie privée. http://www.privacycommission.be/fr.

20. Ayache N, Clatz 0 , Delingette $\mathrm{H}$, et al. Vers un patient numérisé personnalisé pour le diagnostic et la thérapie guidés par l'image. Med Sci (Paris) 2011 ; $27: 208-13$.

\section{TIRÉS À PART}

S. Van Sint Jan 